\title{
The polymer composites' morphological structure simulation
}

\author{
S. A. Drozdov, V. G. Nazarov, S. A. Nozdrachev, Yu. V. Rudyak, G. O. Rytikov \\ Moscow Polytechnic University, Pryanishnikova st., 2A, Moscow, 127550, Russia \\ sergcuxi@rambler.ru
}

PACS 68.55.Nq

DOI 10.17586/2220-8054-2017-8-1-137-145

\begin{abstract}
The digital two-dimensional Fourier decomposition of surface scanning electron microscopy (SEM) micrographs is used to detect the regular morphological structures on a polymer composite surface. Analyzing the SEM images of fluorinated and sulfonated low density polyethylene (LDPE), we identify and specify both regular and irregular submicron surface structures. The possibility of similar information processing techniques generalization for the morphological simulating of modified polymers bulk structures is discussed.
\end{abstract}

Keywords: Surface and bulk modification of polymers, gas-phase fluorination and sulfonation, surface structure, image processing and analysis, scanning electron microscopy.

Received: 20 July 2016

Revised: 28 November 2016

\section{Introduction}

The polymer composite material is a solid two- or multicomponent system based on polymeric matrix with some fillers and/or ingredients located in a volume or on the surface [1]. The main advantages of such materials include a significant improvement in some physicochemical and mechanical properties compared to the properties of merging the composite material's individual components. The considerable enhancement of the mechanical (deformation, elastic and endurance), thermo- and electrical properties, as well as wettability, permeability, radiation resistance and other properties of polymer composite materials was noted and investigated [2-7].

Recently, additive manufacturing technology for polymer composite synthesis has spread. Some characteristics of polymer matrix surface layers are changed under the chemical, mechanical, thermal or complex influences in the "surface modification" case. The "bulk modification" of polymer matrix is implemented as the formation of inclusions, cracking, chemical heterogeneities, etc. and used mostly at the stage of polymerization. However, additive technologies in the "bulk modification" case are also applicable [8-17].

The influence of the surface and bulk morphology of a composite on its' chemical, mechanical, thermal and other properties has been repeatedly noted [18-25]. Great attention was not only paid to the chemical composition of the polymer matrix, modifiers and intensity of interfacial interaction, but also to the morphological structure of the surfaces and volumes of the objects under consideration when describing the properties of polymer composite materials $[9,16,21]$. The role of surface and bulk morphology was mainly observed at the qualitative level and was followed by a demonstration of images received by means of electronic, optical near-field, atomic force and other special microscopy [18,25,28-30].

Mathematical function of the profile cross sections of the considered sample [2], some collective integrated generalized characteristics (waviness, roughness, etc) $[31,32]$ or the empirical distributions on any parameters [25, $33,34]$ were used for the qualitative description of regular structures' shapes. However, the applied methods revealing the localization of inhomogeneities and techniques to construct the corresponding empirical distributions in the reviewed articles were not published.

The present work is devoted to the development of an approach for the description of topographic and stereometric properties of polymer composites obtained in various ways. It is anticipated that the approach will allow comparison of the morphological structure of the materials and, to some extent, to analyze the relationships between individual physicochemical properties and characteristics of the detected structures. The task of building the integrated structural-functional models of developed polymer composites is very important, because experiments to develop new polymer composite materials which possess a set of defined physicochemical properties are often quite expensive and quite complex technically. 


\section{The experimental data}

Some different polymer composites had their physical properties investigated (e.g. wettability, permeability, strength, etc.) and their surfaces were subjected to imaging [35-38].

The images of surfaces of polymer composites made on the basis of low density polyethylene (LDPE) resulting from $\sim 30$ minutes sulfonation and $\sim 15$ minutes fluorination are presented in Fig. 1a and Fig. 1b, respectively.

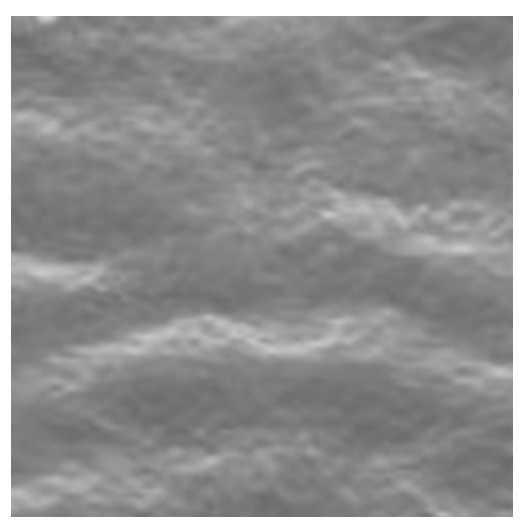

a) sulfonated sample

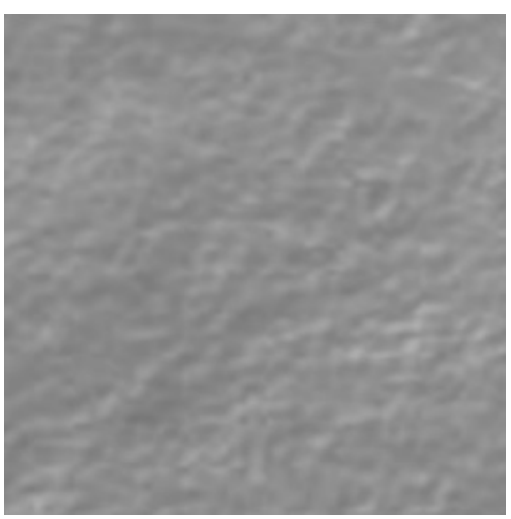

b) fluorinated sample

FIG. 1. SEM-micrographs of the samples' surfaces for the a) sulfonated and b) fluorinated samples

The images were reduced to the same scale for standardization of the data processing procedures. Further digital processing was subjected to square sub images of 128 by 128 pixels. The typical pixel size for the presented images was $\sim 0.04 \mu \mathrm{m}$. Digital images of the surfaces of the samples are characterized by horizontal and vertical resolution. The horizontal resolution here is the number of pixels per unit length (e.g., 300 dpi is 300 pixels per inch). Vertical resolution here means the maximum number available in the used encoding format of image gradation values of pixels' brightness (luminance for displays or optical density for printing stamps). The vertical resolution of represented images is 8 bits, which corresponds to 256 possible levels of pixel brightness (from 0 ("black") to 255 ("white") with increment of 1). And "unit" change of pixel's brightness corresponds to $\sim 2 \mathrm{~nm}$ changing of the local relief height.

The traditional way of quantitatively describing the surface structures is the construction of empirical probability distributions on any parameters (see, for example, $[18,25,38]$ ). The empirical distributions of the observed non-periodic structures from the amounts of pixels forming them were built taking into account features of digital image presentation formats. "The observed non-periodic structure" here refers to a region of the image, bounded by the closed contour formed by pixels of the same brightness. Such distributions actually coincide with the distributions of the probability of observing a heterogeneous surface structure depending on the square of the projection of this structure on a horizontal plane. This method can be applied when the observed surface features are few, and they are obviously non-periodic [39].

The empirical distributions histograms of quantities of the "observed" structures for the sulfonated and for the fluorinated polymeric samples are presented in Fig. $2 \mathrm{a}$ and Fig. $2 \mathrm{~b}$ respectively.

It is clearly seen that the sulfonated (a) and the fluorinated (b) polymer matrices are characterized by significantly different empirical distributions of their images' pixels' brightness. The empirical distribution of submicron objects for the fluorinated sample is much narrower than for the sulfonated one. The observed fractions of "convex" and "concave" objects are significantly different in case (a) and approximately the same in case (b). This is due to the fact that the sulfonation generates micro-sized cavities on the surface of experimental samples and the fluorination contributes to the increase in its average roughness [37,38].

\section{The surface morphological structures simulating}

\subsection{Concept}

It is obvious that the above method of quantitatively describing the composite surface features does not allow one to restore the morphological structure of the sample surface, and requires considerable effort to ensure the possibility of comparison of results obtained by different research groups (e.g. selection of base level and cutoff level [39] are not standardized and implemented by researchers arbitrarily). We believe that the leading candidate for a unified way of constructing morphological patterns of the sample surfaces is their representation in the form 


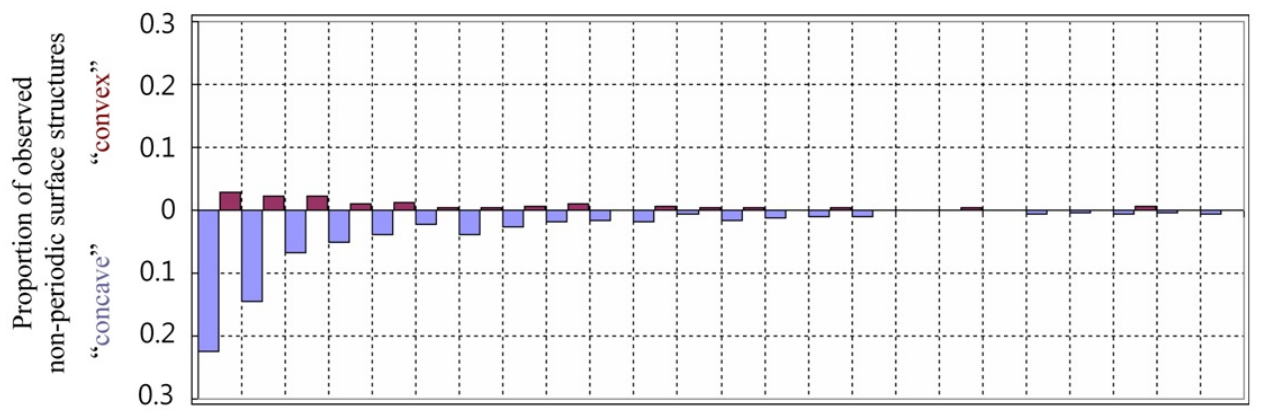

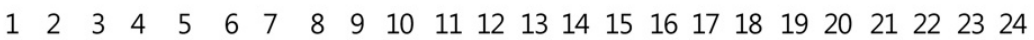

Number of pixels forming structures' images

a) sulfonated sample

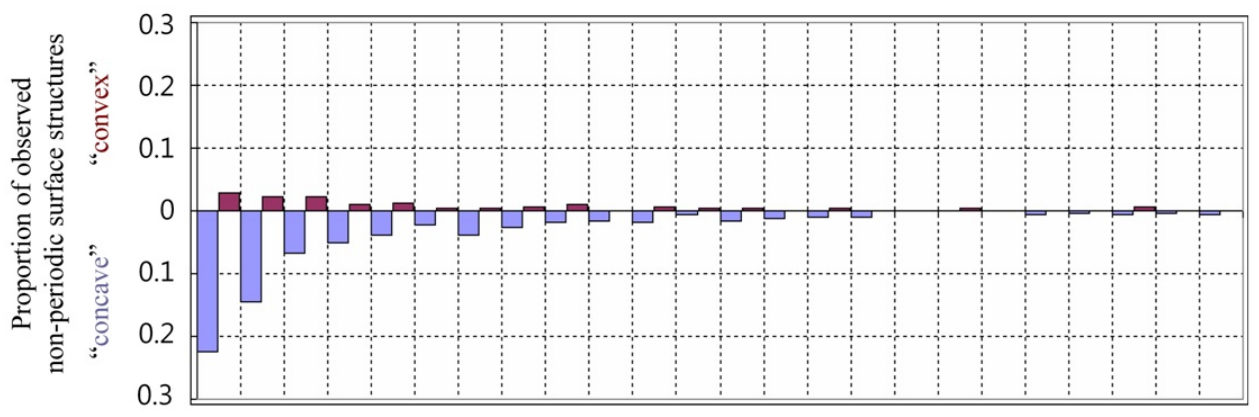

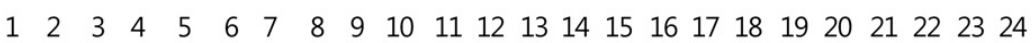

Number of pixels forming structures' images

b) fluorinated sample

FIG. 2. The empirical distributions of the "observed" structures for the a) sulfonated and b) fluorinated samples

of a linear combination of any known two-dimensional orthogonal functions forming a basis. As an example, we have used the two-dimensional Fourier decomposition of digital images of the corresponding samples.

The well-known relations were applied for the two-dimensional Fourier decomposition:

$$
\begin{aligned}
& f(x, y)=\sum_{k, l=0}^{\infty} \sum_{j=1,2,3,4} a_{j k l} \cdot \varphi_{j k l}(x, y), \\
& \varphi_{j k l}(x, y)=\left\{\begin{array}{c}
\sin \\
\cos
\end{array}\right\} \frac{k \cdot 2 \pi x}{L_{x}}\left\{\begin{array}{c}
\sin \\
\cos
\end{array}\right\} \frac{l \cdot 2 \pi x}{L_{y}}, \\
& a_{j k l}=\frac{2^{\lambda}}{L_{x} L_{y}} \int_{0}^{L_{x}} \int_{0}^{L_{y}} f(x, y) \cdot \varphi_{j k l}(x, y) d x d y,
\end{aligned}
$$

where $L_{x}, L_{y}$ - the spatial periods "along" two arbitrary orthogonal axes of the coordinate system of sample; $k, l-$ the indexes of the corresponding harmonics, and carried out the necessary numerical integration; $j$ - enumerates all possible combinations of sine and cosine and:

$$
\lambda=\left\{\begin{array}{c}
0 ; k=l=0 \\
1 ; k=0, l \neq 0 \text { or } k \neq 0, l=0, \\
2 ; k \neq 0 \text { and } l \neq 0 .
\end{array}\right.
$$

In this way, a one-to-one correspondence can be established between any flat image $f(x, y)$ and its twodimensional spatial spectrum, where the two-dimensional Fourier amplitudes $A_{k l}=\sqrt{\sum_{j=1}^{4} a_{j k l}^{2}}$ are the discrete functions of $k, l$ indexes. 


\subsection{Implementation}

It is readily obvious that matching the real digital image to the set of the decomposition's amplitudes requires one to make a number of assumptions and approximations:

1) Summarizing in (1) cannot be carried out in an infinitely large number of terms;

2) Integration in (1) is carried out by numerical methods, i.e. approximately.

Actually, used methods of numerical integration indicate the minimum number of sequence values that are necessary for numerical calculations of varying amplitude decay with reasonable accuracy. We considered the simplest methods (Simpson's and trapezium rule) of numerical integration. The relative error of numerical integration for some simple model functions (represented by 5 points per period) amounted to $\sim 5 \%$. And for 7 points per period, the relative error amounted to $\sim 0.5 \%$, which is less than the "instrument" relative error $\sim 0.7 \%$ estimated as the reciprocal average value of pixels' brightness.

Thus, we were limited to 8 points representation of the spatial period "with a reserve". This is more restrictive than what is prescribed by the Kotelnikov theorem, but it provides a guaranteed accuracy of numerical integration and greatly improves the speed of calculations. Thus, the maximum spatial frequency of the "calculated" harmonics was defined as $2 \pi \cdot 8 / L$, which corresponds to the maximum index values $k_{\max }=l_{\max }=L / 8$. Therefore, if the spatial period is selected as 128 units (as in the example), the summarizing should be carried out for 16 two-dimensional harmonics.

In our case, the spatial reference periods are characterized by geometric sizes of the selected subareas and match ( $\left.L_{x}=L_{y}=128\right)$. The calculated "top-left realization" spectra of two-dimensional surfaces images of the fluorinated and sulfonated samples are presented in Fig. 3.

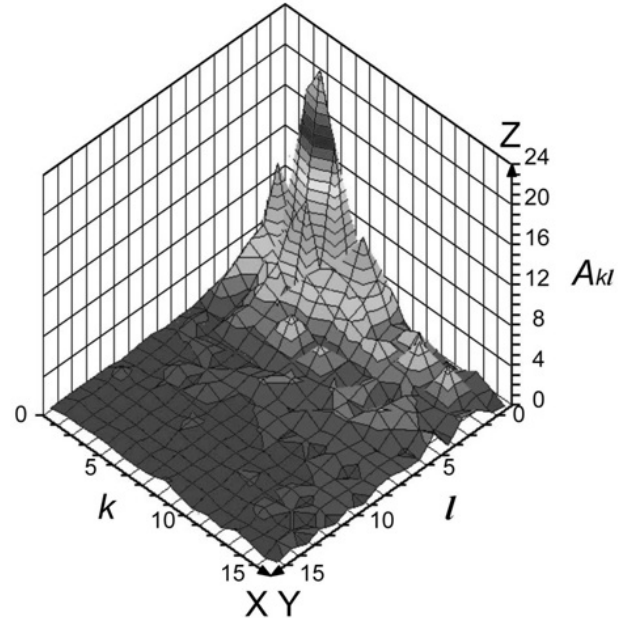

a) sulfonated sample

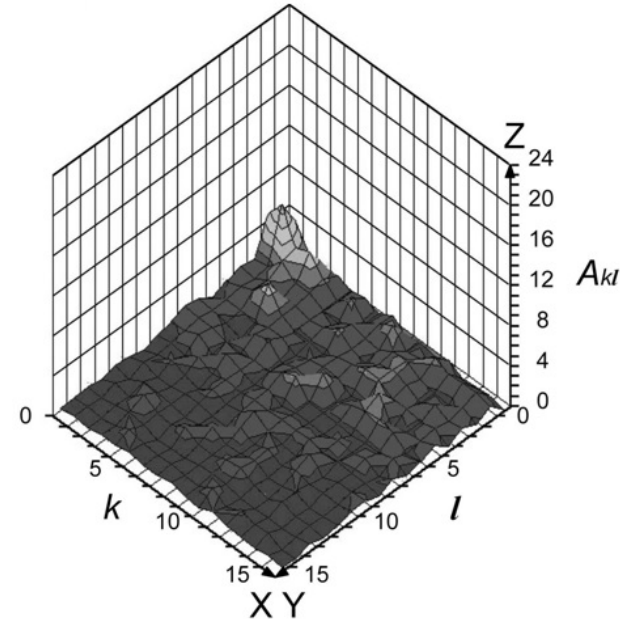

b) fluorinated sample

FIG. 3. The particular morphological spectra for the: a) sulfonated and b) fluorinated samples

The representation of the surface in the form of an expansion for any system of periodic (or quasiperiodic) of orthogonal functions leads to the following interpretation of the notion "regular surface structure": each amplitude of a certain basis function corresponds to one of a two-dimensional periodic lattice with two spatial periods. Then, the total number of observed regular structures is determined by the geometric size and horizontal resolution of the analyzed images. This means that at a given resolution the fixed geometric size of the image sample is mapped to a finite set of two-dimensional periodic lattices. Moreover, the smaller the geometric dimensions of the sample, the smaller the number of two-dimensional periodic lattices can be identified through analysis of the image.

To reduce the degree of dependence of the result from the geometric dimensions and resolution of the analyzed images, it is necessary to apply the classical methodology of mathematical statistics, i.e., to solve the problem of statistical reliability of the obtained results.

\subsection{Identification}

Scanning the whole image with a sub-region of 128 by 128 pixels we got the set of particular morphological spectra (see Fig. 3). There were calculated the average values (Fig. 4), the standard deviations and the t-statistics for each of received 2D-Fourier amplitudes. 


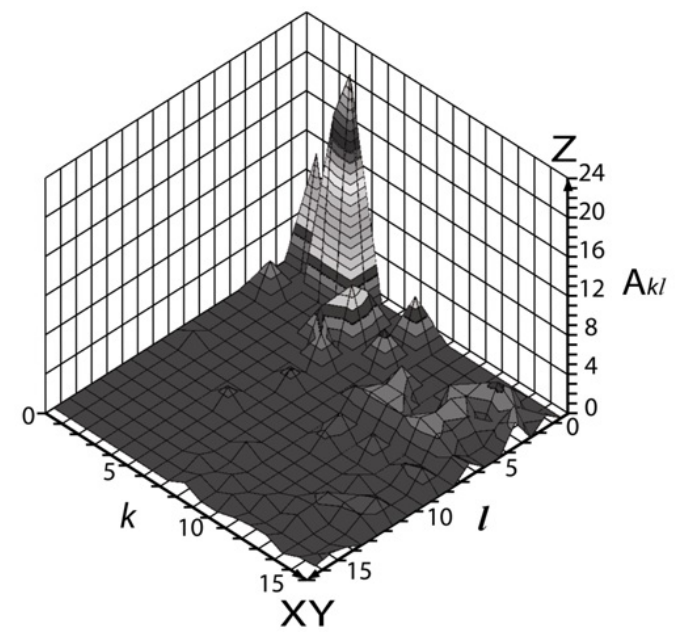

a) sulfonated sample

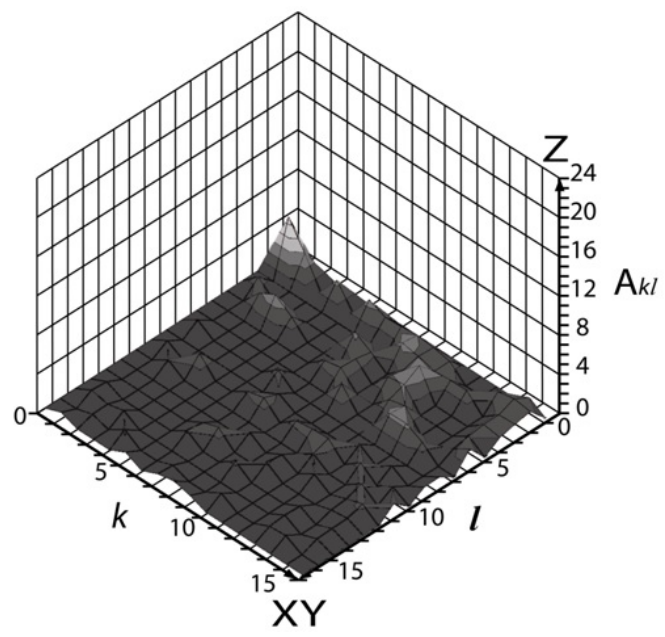

b) fluorinated sample

FIG. 4. The average morphological spectra for the: a) sulfonated and b) fluorinated sample

The set of the calculated amplitudes was divided into the statistically significant and statistically insignificant components using Student's t-test. The amplitude is statistically significant if its empirical t-statistics exceed the critical value dictated by the Student's distribution. The parameters of Student's distribution are "the error probability" and "the number of degrees of freedom". "The error probability" means the probability of rejecting the null hypothesis about the statistical significance of the estimated factor when using bilateral Student's distribution. "The number of degrees of freedom" is defined as the difference between the number of experimental observations and the number of coupling equations.

According to our concept, the statistically significant amplitudes form a model of the morphological spectrum of the surface, and the statistically insignificant ones - the model of morphological noise. We aim to use the statistically significant "average" Fourier amplitudes for quantitative comparison of surface morphologies (and, in the future, of the bulks) of experimental samples (for example, when studying the dependence of the surface micro relief from the duration and the nature of the processing chemicals).

The task of identifying the surface structures and the task of digital modeling/restoring ones (under the "average" morphological spectrum) are characterized by different values of "the error probability" in Student's criteria. It is necessary to choose the minimum (acceptable for the researcher) value of "the error probability" $\alpha$ (e.g., $0.0001,0.05$ etc.) for a reliable identification of the most significant structures. The structures of morphological spectra of sulfonated and fluorinated samples (in cases of the relevant two-dimensional lattices' identification "reliabilities", $(1-\alpha)$, are 0.9999 and 0.95 respectively) are shown in Fig. 5 schematically.

Obviously, the more reliable the obtained (Fig. 4) amplitudes are, the smaller number of them involves 2DFourier morphological spectrum (Fig. 5a) and Fig. 5b. We believe that such surface properties as, for example, wettability determined by small number of statistically significant two-dimensional periodic lattices which form the "effective" microrelief. But when reconstructing (and simulating) the surface (see section 3D), a greater number of amplitudes (Fig. 5c) and (Fig. 5d) with a smaller (but adequate) statistical significance (probability of error $\sim 0.05$ ) provide higher quality for matching the models with the images of the experimental samples' surfaces.

\subsection{Simulation}

The additional information about the Fourier spectra harmonics phases (along with Fourier spectra harmonics amplitudes) is necessary for realistic simulation of the samples' surfaces. This information is contained in $a_{j k l}$ values used to calculate each amplitude $A_{k l}$. Thus, the "phase" information for all statistically significant harmonics was taken into account when restoring of the surface under the average spectrum and statistically insignificant harmonics were ignored.

In this case, a measure of model quality is the correlation coefficient (or coefficient of determination) between the digital images of the original and the model images. Therefore, the error probability when using the Student's criterion should be selected from the range of researcher-acceptable values (for example, not more than 0.05), but with the need to maximize the value of the correlation coefficient. Otherwise, the excessive demands for statistical 


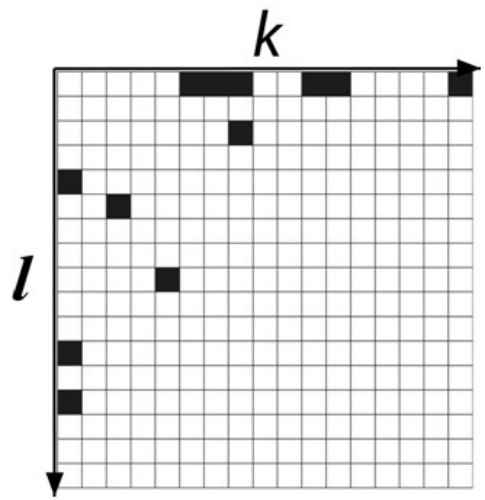

a) sulfonated, 0.9999

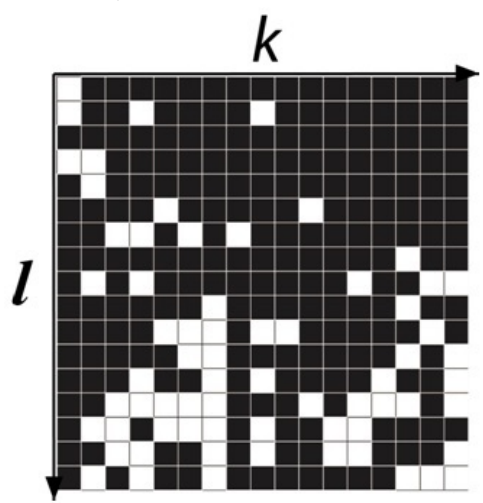

c) sulfonated, 0.95

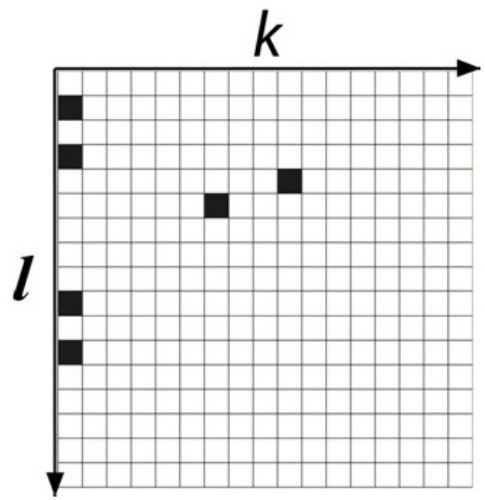

b) fluorinated, 0.9999

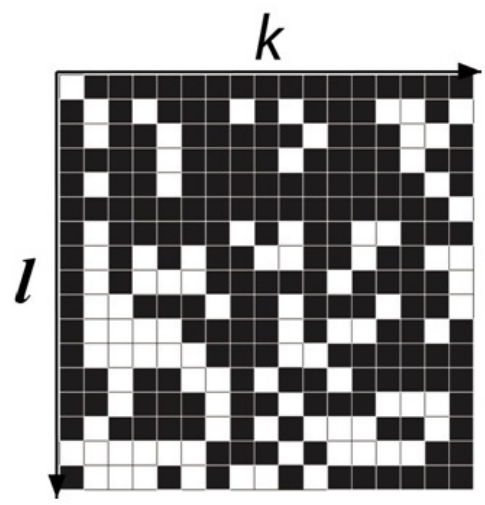

d) fluorinated 0.95

FIG. 5. The morphological spectra's structure for the sulfonated (left) and fluorinated (right) samples with 0.9999 (top) and 0.95 (bottom) reliabilities

significance of the identified surface structures hamper the maintenance of an acceptable quality reconstruction (or modeling) of samples' surfaces under the average morphological spectrum.

The two-dimensional digital relief maps of samples' surfaces and their morphological models are shown in Fig. 6.

When comparing digital originals and morphological models (the error probability 0.05 ) of images of the fluorinated and sulfonated experimental samples surfaces, the correlation coefficient was estimated to be $\sim 0.83$ and $\sim 0.92$, respectively, indicating acceptable quality of the obtained models and the adequacy of this method for solving problems of the polymeric composites surfaces' characterization.

The results of the surfaces' image reconstruction under the average two-dimensional Fourier spectra with 0.9999 and 0.95 harmonics reliabilities are represented in the table (reliability defines as $(1-\alpha)$, where $\alpha-$ "Student's" error probability).

TABLE 1. The surfaces' images restoring quality

\begin{tabular}{|c|c|c|c|c|}
\hline & \multicolumn{2}{|c|}{ The sulfonated sample } & \multicolumn{2}{c|}{ The fluorinated sample } \\
\hline The reliability & 0.9999 & 0.95 & 0.9999 & 0.95 \\
\hline The number of significant amplitudes, units & 67 & 244 & 96 & 250 \\
\hline The proportion of significant amplitudes, \% & 26 & 95 & 37.5 & 98 \\
\hline The correlation coefficient, units & 0.67 & 0.86 & 0.52 & 0.8 \\
\hline
\end{tabular}

In the 0.9999 reliability case for the sulfonated sample, there are 67 statistically significant amplitudes (i.e. $\sim 26 \%$ of the total number; see Fig. 5). In the 0.95 reliability case for the sulfonated sample there are 244 statistically significant amplitudes ( $\sim 95 \%$ from total number). The rest of the two-dimensional Fourier spectrum amplitudes form the "morphological noise". The correlation coefficients for the pixels' brightness "between the 


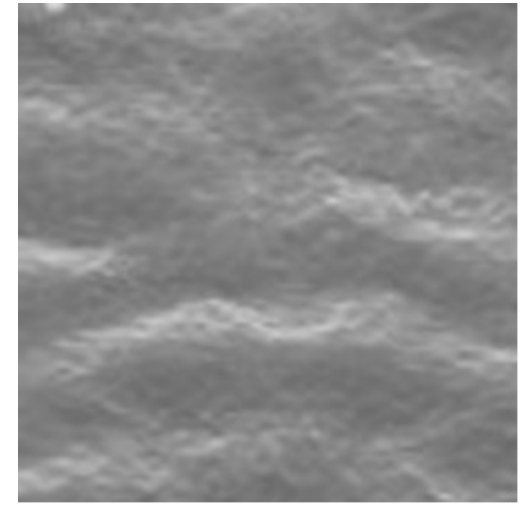

a) sulfonated sample

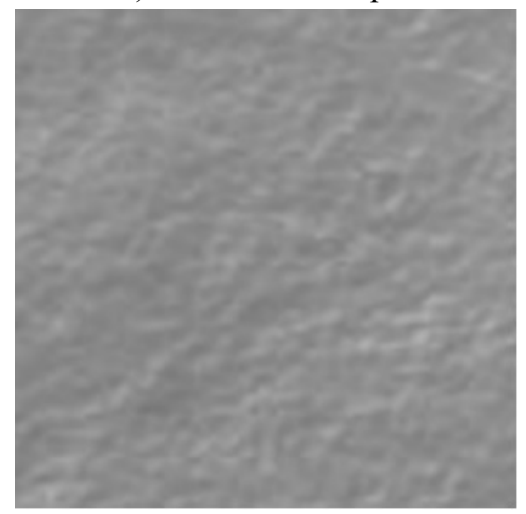

d) fluorinated sample

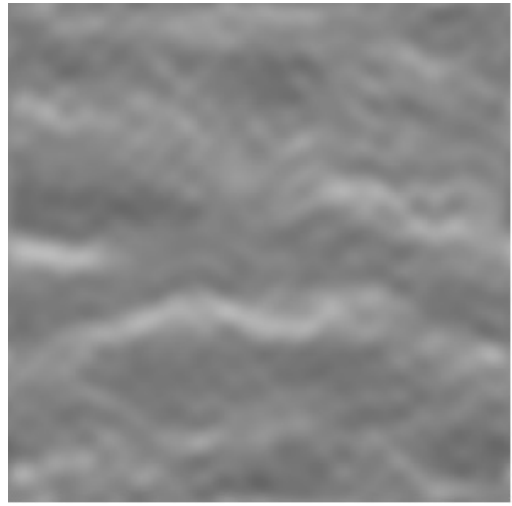

b) 0.95 reliability

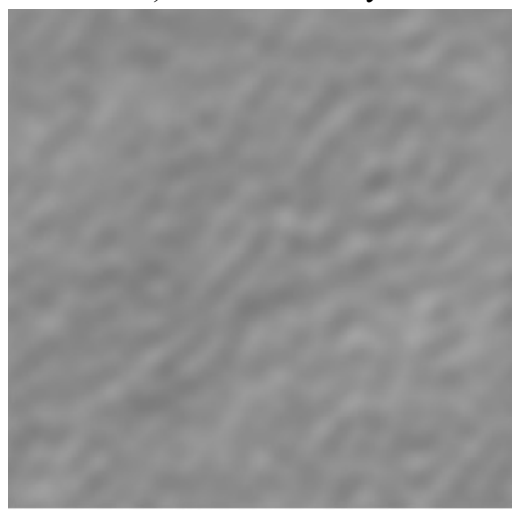

e) 0.95 reliability

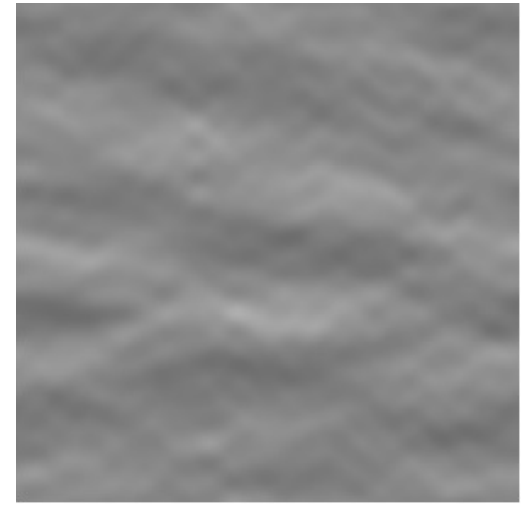

c) 0.9999 reliability

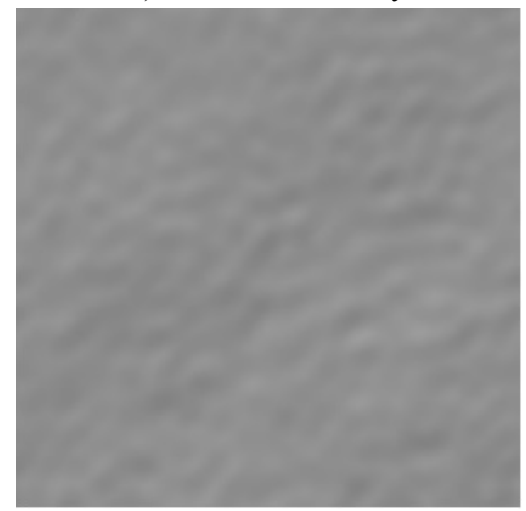

f) 0.9999 reliability

FIG. 6. The two-dimensional digital relief maps of the sulfonated and fluorinated samples surfaces and their reconstructions with different reliability values

samples and the models" are $\sim 0.67$ and $\sim 0.86$ for 0.9999 and 0.95 reliability cases respectively. Similarly, for the fluorinated sample: the 0.9999 reliability corresponds to $96(37.5 \%)$ significant amplitudes, the 0.95 reliability to $250(98 \%)$ significant amplitudes, and provides 0.52 and 0.8 correlation coefficient values respectively.

Thus, the greater reliability in morphological spectrum amplitudes identification is required by the researcher, the smaller number of the periodic lattices (forming the surface relief) will be identified and the worse quality of surface restoring will be achieved. Conversely, the greater the quality of correspondence between reconstructed and initial images is required, the less the accuracy in the morphological spectrum identification it's necessary to settle for.

\section{The volume morphological structure modeling (concept)}

It is proposed to use a technique similar to that described above for the simulation of the volume morphological structure of the modified polymer. Since the volumetric modification is the formation of submicron pores, cavities, inclusions and impurities (chemically homogeneous or chemically heterogeneous with respect to the original polymer matrix), it is necessary to identify three-dimensional regular structure (three-dimensional periodic lattices) the superposition of which would form the corresponding volumetric heterogeneity. 
Three-dimensional Fourier decomposition is determined by:

$$
\begin{aligned}
& \rho(x, y, z)=\sum_{k, l, m=0}^{\infty} \sum_{j=1, \ldots, 8} a_{j k l m} \cdot \varphi_{j k l m}(x, y, z), \\
& \varphi_{j k l m}(x, y, z)=\left\{\begin{array}{c}
\sin \\
\cos
\end{array}\right\} \frac{k \cdot 2 \pi x}{L_{x}}\left\{\begin{array}{c}
\sin \\
\cos
\end{array}\right\} \frac{l \cdot 2 \pi x}{L_{y}}\left\{\begin{array}{c}
\sin \\
\cos
\end{array}\right\} \frac{m \cdot 2 \pi x}{L_{z}}, \\
& a_{j k l m}=\frac{2^{\lambda}}{L_{x} L_{y} L_{z}} \int_{0}^{L_{x}} \int_{0}^{L_{y}} \int_{0}^{L_{z}} \rho(x, y, z) \cdot \varphi_{j k l m}(x, y, z) d x d y d z .
\end{aligned}
$$

Here, $L_{x}, L_{y}, L_{z}$ - the spatial periods "along" three arbitrary orthogonal axes of the coordinate system of sample; $k, l, m$ - the indices of the corresponding harmonics, and

$$
\lambda=\left\{\begin{array}{l}
0 ; k=l=m=0 \\
1 ; k=l=0, m \neq 0 \text { or } k=m=0, l \neq 0 \text { or } l=m=0, k \neq 0 \\
2 ; k=0, l \neq 0, m \neq 0 \text { or } k \neq 0, l=0, m \neq 0 \text { or } k \neq 0, l \neq 0, m=0 \\
3 ; k \neq 0, l \neq 0, m \neq 0
\end{array}\right.
$$

Thus, any empirical three-dimensional distribution of density of matter $\rho(x, y, z)$ can be mapped to morphological range $A_{k l m}=\sqrt{\sum_{j=1}^{8} a_{j k l m}^{2}}$, allowing you to visualize the morphological model in the form of modulation of optical density, using the color space or in the form of animation. We consider the possibility of practical implementation of these methods for visualizing morphological patterns of volumetric modified polymers as an important task for further research.

\section{Conclusion}

This paper discusses ways to identify morphological structures on the surfaces and in the volumes of polymer composites. Examples of surface-modified polymers obtained by gas-phase sulfonation and fluorination were considered. A number of regular surface structures were revealed when processing was obtained by means of scanning electron microscopy images of samples' surfaces with two-dimensional Fourier analysis. A method based on three-dimensional Fourier analysis of the distribution of density fluctuations of matter for the identification and specification of the regular submicron structures in polymeric composites volumes was offered.

\section{Acknowledgements}

The work was performed with financial support from the RFBR grant 16-03-00540. The authors express their deep appreciation to the referee and the editor for helpful and significant suggestions and tips.

\section{References}

[1] Kerber M.L., Vinogradov V.M., Golovkin G.S., et al. Polymer composite materials: structure, properties, technology. SPb.: Profession, 2008. (RUS)

[2] Myakin S.V., Sychev M.M., Zahradnicek A.L., Vasilyev V.I., Sosnov E.A., Kolovangina E.S. Investigation of radiation resistance of polyimide films under the influence of high doses of accelerated electrons. Bulletin of St. Petersburg state technological Institute (technical University), 2012, 14, P. 36-40. (RUS)

[3] Sevast'yanov V.I., Nemets E.A., Stolyarov V.P., Baranov V.A., Bozhko N.N., Nazarov V.G. omparative study of the influence of polyethylene film surface modification on interaction with blood components. Inorganic Materials: Applied Research, 2011,2 (2), P. 46-152.

[4] Chung J.Y., Stafford C.M. and Youngblood J.P. Anisotropic wetting on tunable micro-wrinkled surfaces. Soft Matter., 2007, 3 (9), P. $1163-1169$

[5] Pertsov A.V., Soboleva O.A., Protsenko P.V. and Nazarov V.G. Selective wetting in a hydrocarbon liquid-perfluorocarbon liquid-solid system. Colloid Journal, 2008, 70 (6), P. 759-762.

[6] Nazarov V.G., Stolyarov V.P., Volynskii A.L., Yarysheva L.M., Bakeev N.F. Transformation of the modified layer of fluorinated polyolefins under stretching. Polymer Science. Series A, 2012, 54 (9), P. 679-683.

[7] Arzhakova O.V., Dolgova A.A., Yarysheva L.M., Volynskii A.L. and Bakeev N.F. Development of a Stable Open-Porous Structure in the Solvent-Crazed High-Density Polyethylene. Inorganic Materials: Applied Research, 2011, 2 (5), P. 493-498.

[8] Nazarov V.G. Multiple surface structures in polyolefins formed by modification methods. Journal of Applied Polymer Science, 2005, 95 (5), P. 1198-1208.

[9] Volynsky A.L., Kechekjan A.S., Bakeev N.F. Polymer-polymer systems "rigid coating on a compliant base". Polimer Science. Series A, 2003, 45 (7), P. 1130. (RUS) 
[10] Goikhman M.Ya., Subbotina L.I., Romashkova K.A., Smirnov N.N., Ershov Y.A., Gofman I.V., Lukoshkin V.A., Yakimansky A.V., Kudryavtsev V.V. Synthesis and properties of copolymers of glycidyl methacrylate with side chromophore groups. Polimer Science. Series A, 2007, 49 (7), P. 1170-1180. (RUS)

[11] Belomoina N.M., Rusanov A.L. Modified polymers and materials on their basis. Polimer Science. Series A, 2007,49 (7), P. $1426-1447$. (RUS)

[12] Badamshina E.R., Gafurova M.P. Modification of properties of polymers by doping with fullerene C60. Polymer Science. Series A, 2008, 50 (8), P. 1572-1584. (RUS)

[13] Valuev I.L., Valuev L.I., Obydennova V. I., Sytov G.A., Vanchugov L.V. Modified polyurethanes: a new type thromboresistant polymers. Polymer Science. Series A, 2010, 52 (8), P. 1432-1435. (RUS)

[14] Panin S.V., Kornienko L.A., Puvadin T., Sergeev V. P., Ivanova L.R., Shilko S.V. The modification of ultra-high molecular weight polyethylene with high-energy surface treatment methods. Advanced materials, 2011, 13, P. 376-383. (RUS)

[15] Hodyrevskaya Y.I., Tverdokhlebov S.I., Kudryavtseva Y.A. Plasma-chemical modification of polymer materials for cardiovascular surgery to control the degree of wettability. Bulletin of the Tomsk Polytechnic University. Mathematics and mechanics. Physics, 2014,325 (2), P. 158-165. (RUS)

[16] Nazarov V.G. Structure and composition of the surface layer in polymers modified by elemental fluorine. Journal of Applied Polymer Science, 2005, 95 (4), P. 897-902.

[17] Nazarov V.G., Kondratov A.P., Stolyarov V.P., Evlampieva L.A., Baranov V.A., Gagarin M.V. Morphology of the surface layer of polymers modified by fluorine gas. Polymer Science. Series A, 2006, 48 (11), P. 1976-1985. (RUS)

[18] Krisilova E.V., Eliseeva T.V., Grechkina M.V. The analysis of the structure of the surface sulfocationites perfluorinated membranes in hydrogen and lysine forms by atomic force microscopy. Sorption and chromatographic processes, 2010, 10(1), P. 103-107. (RUS)

[19] Safronova T.V., Putlyaev V.I. Medical inorganic materials science in Russia: the calcium-phosphate materials. Nanosystems: physics, chemistry, mathematics, 2013, 4 (1), P. 24-47. (RUS)

[20] Kurylenko K.A., Brylev O.A., Filippov T.V., Baranchikov A.E., Shlyakhtin O.A. Cryogenically synthesis of cathode materials based on $\mathrm{LiNi}_{0.4} \mathrm{Mn}_{0.40 .2} \mathrm{O}_{2}$ for Li-ion batteries. Nanosystems: physics, chemistry, mathematics, 2013, 4 (1), P. 105-112. (RUS)

[21] Shipovskaya A.B., Rudenko D.A., Fomina V.I. and Ostrovsky N.V. Structure and Properties of Chitosan-Based Films for Biomedical Purposes. European Journal of Natural History, 2012, 6, P. 7-12.

[22] Volynskii A.L., Panchuk D.A., Bolshakova A.V., Yarysheva L.M., Bazhenov S.L., Yablokov M.Y., Gilman A.B. and Bakeev N.F. The structure and properties of thin aluminum coatings. Thin Solid Films, 2013, 536, P. 179-186.

[23] Volynskii A.L., Yarysheva L.M., Bagrov D.V., Rukhlya E.G., Yarysheva A.Y., Bakeev N.F., Nazarov V.G., Stolyarov V.P. and Tsypysheva S.V. Evaluation of the deformation strength properties of a fluorinated polymer layer on the surface of poly(ethylene terephthalate) films. Doklady Physical Chemistry, 2012, 442 (1), P. 5-7.

[24] Sotskaya N.V., Dolgikh O.V., Kashkarov V.M., Lenshin A.S., Kotlyarova E. A., Makarov S.V. Physico-chemical properties of surfaces modified with nanoparticles of metals. Sorption and chromatographic processes, 2009, 9 (5), P. 643-652. (RUS)

[25] Vernigorov K.B., Alentiev A., Meshkov I.B., Muzafarov A.M., Voronina E.N., Novikov L.S., Chernik V.N. Improving the sustainability of the thermoplastic polyimide exposure to atomic oxygen by modification of the hyperbranched polyethoxysiloxanes. Advanced materials, 2011, 4, P. 10-17. (RUS)

[26] Prudnikov A.M., Linnik A.I., Shalaev R.V., Rumyantsev V.V., Kucherik A.O., Alodjants A.P., Arakelian S.M. The features of formation and modification of nanostructured films of carbon nitride. Nanosystems: physics, chemistry, mathematics, 2012, 3 (6), P. 134-145. (RUS)

[27] Balakhonov S.V., Luchinkin D.S., Efremova M.V., Churagulov B.R., Tretyakov Yu.D. The synthesis and physics-chemical properties of lithium-doped nanowhiskers on the basis of oxides of vanadium. Nanosystems: physics, chemistry, mathematics, 2011,2 (3), P. 102-112. (RUS)

[28] Goerg H. Michler. Electron Microscopy of Polymers. Springer Laboratory, 2008.

[29] Evdokimov P.V., Putlyaev V.I., Merzlov D.A., Shatalova T.B., Safronova T.V., Klimashin E.S., Churagulov B.R. The polymorphisms of $\mathrm{Ca}_{3}\left(\mathrm{PO}_{4}\right)_{2}$. Nanosystems: physics, chemistry, mathematics, 2013, 4 (1), P. 48-53. (RUS)

[30] Sarychev A.S., Parshina E.Yu., Bayzhumanov A.A., Semenova A.A., Gudilin E.A., Maksimov G.V. The influence of silver hydrosols on structural integrity of erythrocytes. Nanosystems: physics, chemistry, mathematics, 2013, 4 (1), P. 66-71. (RUS)

[31] Manakina D.S., Tcherdyntsev V.V., Ludikova A.A. The investigation of the structure and roughness of the hydrophobic composite coatings on the basis of polysulfone. Modern problems of science and education, 2012, 5, P. 126. (RUS)

[32] Senatov F.S., Maksimkin V.A., Yergin K.S. The investigation of structure of coatings based on polyster by scanning electron microscopy and optical profilometry. Modern problems of science and education, 2012, 5, P. 129. (RUS)

[33] Arzhakova O.V., Dolgova A.A., Yarysheva L.M., Volinsky A.L., Bakeev N.F. The creation of a stable open-pored structure in the high-density polyethylene deformed in liquid media by the mechanism of crazing. Advanced materials, 2011, 1, P. 39-46.

[34] Yanovsky Yu.G., Nikitina E.A., Karnet Yu.N., Valiev H.H., Yumashev O.V., Semenov N.A. Quantum-mechanical description of adhesive interactions and experimental studies of the processes of aggregation of carbon-silicate nanoparticles-reinforcing fillers of polymer composites. Physical Mesomechanics, 2013, 16 (4), P. 85-96. (RUS)

[35] Nazarov V.G., Sevast'Yanov V.I., Stolyarov V.P., Evlampieva L.A., Baranov V.A., Gagarin M.V. Simulation of the development of the mosaic surface structures in polyethylene. Polymer Science. Series B, 2009, 51 (9-10), P. 367-376.

[36] Nazarov V.G., Stolyarov V.P., Evlampieva L.A., Baranov V.A., Gagarin M.V. Simulation of sulfonation and structure of surface layer in polyethylene. Polymer Science. Series A, 2009, 51 (3), P. 340-349.

[37] Nazarov V.G., Stolyarov V.P., Molchanov S.P., Yurasik G.A., Artemenko M.N. Heterogeneous fluorine-containing surface macro-, microand nanostructures in polymer films and their applications. Polymer Science. Series A, 2013, 55 (11), P. $652-665$.

[38] Nazarov V.G., Stolyarov V.P., Novikov M.S., Mikhaleva L.A., Bablyuk E.B., Benda A.F. The influence of fluorination on surface characteristics of polymeric materials. Bulletin of Higher Educational Institutions. Problems of printing and publishing industries, 2011, 2, P. 118-127. (RUS)

[39] Kopachev E.S., Nozdrachev S.A., Petrushin V.N., Rudyak Yu.V., Rytikov G.O., Nazarov V.G. A complex technique of surface image characterization for polymer composites. Physical mesomechanics, 2015, 18 (6), P. 98-110. (RUS) 